\title{
Autoimmune Ocular Myasthenia Gravis: Diagnostic Utility of Autoantibodies to The Neuromuscular Junction in Eye Disorders
}

\author{
Pankaj Kumar ${ }^{1}$ and Hans Frykman ${ }^{2 *}$ \\ ${ }^{2}$ Department of Medicine, University of British Columbia, Vancouver, British Columbia, Canada
}

*Corresponding author: Hans Frykman FRCPC Clinical Assistant Professor, Department of Medicine, University of British Columbia, Vancouver, British Columbia, Canada.
Received Date: September 03, 2020

Published Date: October 23, 2020

Key Words: Myasthenia Gravis (MG), Autoantibodies (Abs), Achr, Musk, LRP4, RIPA, Cbas

\section{Opinion}

Myasthenia gravis (MG) is a neuromuscular disease, mediated by autoantibodies (Abs) that targets functionally important proteins at the postsynaptic neuromuscular junction (NMJ) [1]. MG patients are typically subgrouped according to their clinical symptoms as well as serum Abs profile, which differs significantly in terms of immunopathology, and their distinct response to treatments. Although MG patients can develop a range of symptoms, however, the hallmark of the disease is the fluctuating muscle weakness which is highly variable in severity [1]. Circulating MG Abs can potentially target any skeletal muscle of the body, however, for the majority of patients, initial weakness starts with extraocular muscles (Ocular MG (OMG)), with a classic presentation of unilateral or bilateral drooping of the upper eyelid (ptosis) and rapidly progressive double vision (diplopia) [1,2]. OMG patients typically complain of weakness and heaviness around eyes that worsens as daygoes on. Patients often raise their eyebrows and chin to lift the eyelid in order to see properly. In approximately $15 \%$ of patients, the muscle weakness remains strictly ocular, however, for the majority of the patients (85\%) the symptoms progress to other parts of the body including, facial, neck, bulbar and limb muscle weakness, resulting in generalized MG (GMG), which usually happens within the first 2 years of disease onset $[1,3]$. Respiratory muscles can also be affect ed by GMG [1]. Thus, prompt and accurate diagnosis of OMG patients is critical as the likelihood of generalization can be reduced with early interventions. Progression to GMG is unlikely if the OMG patients do not generalize within the first three years of the disease onset [2].

The immunopathology of MG is highly diverse and strongly associated with the heterogeneity that is observed among different subgroups [4]. Pathogenic antibodies target a number of transmembrane proteins that are necessary to maintain the NMJ architecture [5]. The most common target is acetylcholine receptors (AChR) that AChR Abs (IgG1/IgG3 subclass) either blocks or activates complement cascade that subsequently cause destruction of the postjunctional membrane and increased endocytosis of the receptors. The muscle-specific kinase proteins (MUSK) of the NMJ are the second most common target for Abs attack in MG patients. MuSK Abs are fundamentally different from AChR Abs as they belong to IgG4 subclass, which does not activate complement [6]. MuSK pathogenic Abs binds directly to MuSK protein and blocks the interaction with the lipoprotein-receptor-related protein 4 (LRP4)-agrin complex that is required for AChR clustering at NMJ [6]. Similarly, LRP4 and agrin pathogenic antibodies block the LRP4-agrin signaling, inactivate MuSK, and inhibit AChR clustering at NMJ [5]. With fewer 
receptors, acetylcholine can no longer trigger the action potential from nerve to muscle cells. There are a number of intracellular proteins (including anti-titin and cortactin) that are probably more useful as biomarkers for clinical characteristics in MG patients; however, they are unlikely to play a direct role in MG pathogenesis $[3,7]$. Assaying for various Abs to the NMJ in eye disorders is crucial; however, challenges remain, in part because of the heterogeneous nature of the disease as well as due to poorly defined diagnostic criteria for OMG. For instance, the serum antibodies from clinically suspected OMG patients are initially assayed for the presence of AChR Abs by gold standard radioimmunoprecipitation assay (RIPA). Although the specificity of the test is almost $100 \%$ in all MG types, the sensitivity of the test is lower in ocular MG (around 50\%), which means that almost $50 \%$ of patients would be falsely called negative [5]. In contrast sensitivity of the test is high in GMG patients (80\%). As a reflex test, Abs against MuSK is assayed by RIPA, however, for the majority of OMG patient's anti-MuSK Abs tend to be negative $[1,6]$. It is possible that seronegative OMG patients may have low affinity/low titer Abs that are below the detection limits of RIPA. In fact, most seronegative OMG patients are harbinger of milder disease with better treatment response [2]. In contrast, if the patients with eye disorders tested positive for AChR or MuSK Abs by RIPA, they have a higher likelihood of developing GMG [8]. It is also possible that some seronegative OMG patients develop Abs against other NMJ proteins such as LRP4, agrin, titin, and cortactin. However, currently, it is difficult to ascertain their specificity and direct role in OMG pathogenesis mainly because most ofthe Abs co-exists with AChR and MuSK Abs. Serological testing for anti-LRP4, anti-striational, and anti-cortactin Abs are of particular interest since these antibodies are increasingly detected in classic OMG patients that are responsive to immunotherapies $[9,10]$.

With the advancements in novel pathophysiologically-driven treatment options (for example, Eculizumab ${ }^{\circledR}$ in refractory AChR Abs positive patients and Rituximab ${ }^{\circledR}$ in MuSK Abs positive patients), it is of utmost importance to accurately identify the specific Abs that is causing the disease in OMG patients. To address this, several novel assays and testing algorithms have been developed. One of the major developments in recent years is the introduction of novel live cell-based assays (CBAs) that have a phenomenal ability to detect low titer antibodies in RIPA-negative myasthenia patients [11]. The live CBAs involve expressing clustered AChR antigens in its native form on the HEK293 cell surface through the co-expression of intracellular protein rapsyn. Low affinity AChR Abs binds well to clustered AChR antigens (Clustered AChR Abs). In routine diagnostics settings, the high sensitivity clustered AChR CBAs can typically detect an additional $20 \%$ of cases, most of them with ocular features [11]. The sensitivity and specificity of the CBAs are further increased when both the adult and embryonic forms of the AChR antigens are used, this is particularly beneficial for OMG diagnostics as an autoimmune response to the embryonic gamma subunit of AChR is maintained in adult extraocular muscles [12].
Moreover, for children, the ability to distinguish between congenital and treatment-responsive acquired MG makes the clustered CBAs test a first-line option [11,13-15]. Overall, most clinical studies have confirmed that clustered AChR and/or LRP4 Abs positive patients have higher prevalence of OMG, milder disease severity and better treatment response [11,13-15]. Unfortunately, due to the unavailability of commercial kits, the CBA tests currently are only available in highly specialized laboratories.

It is important to highlight that confounded test results are possible if the patient had received treatments including immunoglobulin (IVIG) and plasma exchange (PLEX) (within 6 weeks of their antibody test) or Rituximab/Eculizumab (within 24 weeks of their test). Therefore, we propose a full "reflex testing," algorithm on the first sample that is collected before any treatment from a suspected OMG patient. Starting with the RIPA assays for AChR Abs, if AChR Abs RIPA is negative, then reflex to MuSK Abs by RIPA. The seronegative OMG sample should be then concurrently tested for high sensitivity clustered AChR Ab, MuSK Ab, LRP4 Ab by CBAs as well as anti-titin $\mathrm{Ab}$ by indirect immunofluorescence and anti-cortactin $\mathrm{Ab}$ by Western blots. We predict that adopting sensitive and accurate testing algorithms will be crucial for considering more effective therapies in OMG patients.

\section{Acknowledgement}

None.

\section{Conflict of Interest}

Authors declare no conflicts of interest.

\section{References}

1. Gilhus NE, Tzartos S, Evoli A, Palace J, Burns TM, et al. (2019) Myasthenia gravis. Nat Rev Dis Primers 5(1): 30.

2. Melson AT, McClelland CM, Lee MS (2020) Ocular myasthenia gravis: updates on an elusive target. Curr Opin Neurol 33(1): 55-61.

3. Gilhus NE, Verschuuren JJ (2015) Myasthenia gravis: subgroup classification and therapeutic strategies. Lancet Neurol 14(10): 10231036.

4. Romi F, Hong Y, Gilhus NE (2017) Pathophysiology and immunological profile of myasthenia gravis and its subgroups. Curr Opin Immunol 49: 9-13.

5. Oger J, Frykman H (2015) An update on laboratory diagnosis in myasthenia gravis. Clin Chim Acta 444: 43-8.

6. Evoli A, Alboini PE, Damato V, Iorio R, Provenzano C, et al. (2018) Myasthenia gravis with antibodies to MuSK: an update. Ann N Y Acad Sci 1412(1): 82-89.

7. Gilhus NE, Skeie GO, Romi F, Lazaridis K, Zisimopoulou P, et al. (2016) Myasthenia gravis - autoantibody characteristics and their implications for therapy. Nat Rev Neurol 12(5): 259-268.

8. Lazaridis K, Tzartos SJ (2020) Autoantibody Specificities in Myasthenia Gravis; Implications for Improved Diagnostics and Therapeutics. Front Immunol 11: 212 
9. Cordts I, Bodart N, Hartmann K, Karagiorgou K, Tzartos JS, et al. (2017) Screening for lipoprotein receptor-related protein 4-, agrin-, and titinantibodies and exploring the autoimmune spectrum in myasthenia gravis. J Neurol 264(6): 1193-1203.

10. Hong Y, Zisimopoulou P, Trakas N, Karagiorgou K, Stergiou C, et al (2017) Multiple antibody detection in 'seronegative' myasthenia gravis patients. Eur J Neurol 24(6): 844-850.

11. Vincent A, Huda S, Cao M, Cetin H, Koneczny I, et al. (2018) Serological and experimental studies in different forms of myasthenia gravis. Ann $\mathrm{N}$ Y Acad Sci 1413(1): 143-153.

12. Horton RM, Manfredi AA, Conti-Tronconi BM (1993) The 'embryonic' gamma subunit of the nicotinic acetylcholine receptor is expressed in adult extraocular muscle. Neurology 43(5): 983-986.
13. Vincent A, Beeson D, Lang B (2000) Molecular targets for autoimmune and genetic disorders of neuromuscular transmission. Eur J Biochem 267(23): 6717-6728.

14. Vincent A, Newsom-Davis J, Newton P, Beck N (1983) Acetylcholine receptor antibody and clinical response to thymectomy in myasthenia gravis. Neurology 33(10): 1276-1282.

15. Vincent A, Rothwell P (2004) Myasthenia gravis. Autoimmunity 37(4): 317-319. 